analysis must always play a significant part in such a project and a further paper analyses the design and interpretation of genetic surveys.

Many genetic factors become manifest through their influence on growth processes as also do such selective forces as malnutrition and disease. It is to studies of these that the third contribution is directed.

The capacity for physical work is significant as a partial measure of the biological efficiency of a population, and the fourth contribution to the symposium reviews the relatively restricted amount of information at present bearing on this factor.

The next twelve papers deal with studies of physiological adaptation and of genetic variability in populations from Africa, America, Asia, Australia and the circumpolar regions, while the two final papers bear on fascinating projects relating to adaptability in high altitude populations.

The result is a volume of more than five hundred pages which contains, not only scholarly surveys, but also a mass of detailed factual data of much significance.

Even so, cursory comparison between the contributions relating to populations from different regions of the world indicates forcefully the extent of the information that remains to be collected. But such realisation, in no sense diminishes admiration for the body of work already completed. In the words of Sir Lindor Brown in his foreword to the volume, the International Biological Programme has passed its first inertial stages and "a few rocks and sandbanks of solid achievement are beginning to be visible". In throwing these into sharp relief, the present volume cannot fail to stimulate enthusiasm for their further accretion and eventual amalgamation.

E. H. Ashton.

\title{
A PRIMER OF GENETIC METHODOLOGY
}

GENETIC ANALYSIS. W. K. Baker. Riverside studies in Biology. Thomas Nelson, London, 1965. Pp. $x+164$. 15s.

In the preface the author makes the point that it has become increasingly unsatisfactory during the last 20 years or so to divide biology into artificial and exclusive compartments called zoology, botany, genetics, etc. Modern biological research is now concentrating on fundamental problems which require a wide spectrum of techniques and experimental organisms for their elucidation. Genetical techniques for example are central in the investigation of such problems as growth and differentiation although these phenomena encompass a good deal of the whole field of biology. Accordingly, the author has written an explanatory account of some of the more important genetical techniques and the kind of answers they provide to certain biological questions.

The general idea of illustrating the genetical approach and giving details of techniques applied to specific problems is a good one. Also the actual choice of topics to be covered within the compass of a small book is good. Chapters of varying length are devoted to: Introduction to genetic analysis; Genetic symbols and ratios in inheritance; Genetic recombinants; Gene action; Mutation; Cytology and analysis of normal and aberrant chromosomes; the Genetic code and finally Cellular Differentiation. There is also 
a useful appendix which gives a brief description of the life cycles of Drosophila, Neurospora, Escherichia coli and Maize.

Let us look at some of the chapters more closely. The introduction to genetic analysis presents the problem and some of the elementary facts of inheritance in terms of a physicists "black box" type of illustration. Moreover, a new term "genophore" is introduced into a field already overloaded with not generally understood jargon. However well intentioned the whole business is too fancy by half. Sooner or later anyone who wishes to understand something of biology must master the elementary rules of inheritance. Surely the study of a straightforward account of gene segregation in Drosophila-or even peas-is the least painful method. This chapter is sheer waste in a small book.

In the section on gene action the cistron is well defined including the occurrence of intra-cistron complementation. The discussion of the basis of intra-cistron complementation is feeble. The few pages on mutation are almost worthless. They contain a pointless attempt at defining and measuring units of mutation, "mutons". There is no mention of the nucleotide base as the nearest thing to a basic unit of mutation or of the ways in which chemical mutagenesis in increasing our understanding of mutation.

There is an accurate but far too short account of bacterial conjugation and transduction. The description of some of the aspects of the work on the genetic code and protein synthesis is quite good although rather sadly out of date. The significance of such work for our understanding of cellular differentiation is briefly discussed.

A third of the book deals with the analysis and cytology of chromosomes and this is well done. It is difficult to understand why in a book of this nature such a disproportionate amount of space is given to quite sophisticated and specialist aspects of chromosome analysis. Yet other extremely important topics are treated with a brevity which destroys much of their value.

In summary, most sections of this book are fairly accurate, up to date and well presented. In general the relative balance of the subjects dealt with is poor with the result that the majority of chapters are too brief. The printing and illustrations are clear. At I 5 s. this book is reasonable value if read together with one or more other genetic texts of similar standard,

\section{J. A. Pateman.}

\section{INDETERMINATE DETERMINATION !}

SEX DETERMINATION (1st Edition). Guido Bacci. International Series of Monographs in Pure and Applied Biology, Zoology Division vol. 26. Pergamon Press, 1965. Pp. 306. $84 s$.

Professor Bacci's book is a well-conceived and comprehensive account of the facts of sex-determination. The general layout of the material is also commendable for a vast array of material has been organised into a meaningful order. The book is, however, far less satisfactory in its attempts to rise above the facts. The reasons for this are twofold.

First, the sexual systems and cycles which form the basis of the book find significance only when interpreted in genetical terms. And from his first excursion into the genetical underworld the author is clearly in difficulty. Thus his appreciation of the process of meiosis, so basic to the whole issue, 